\title{
Theranostics
}

Commentary

2011; $1: 274-276$

\section{Targeted Nanoprobes Reveal Early Time Point Kinetics In Vivo by Time-Resolved MRI}

\author{
Ashwinkumar Bhirde, Ning Guo, Xiaoyuan Chen ${ }^{凶}$ \\ Laboratory of Molecular Imaging and Nanomedicine (LOMIN), National Institute of Biomedical Imaging and Bioengineer- \\ ing (NIBIB), National Institutes of Health (NIH), Bethesda, Maryland 20892 \\ $\bowtie$ Corresponding author: Xiaoyuan Chen, shawn.chen@nih.gov \\ (C) Ivyspring International Publisher. This is an open-access article distributed under the terms of the Creative Commons License (http://creativecommons.org/ \\ licenses/by-nc-nd/3.0/). Reproduction is permitted for personal, noncommercial use, provided that the article is in whole, unmodified, and properly cited.
}

Received: 2011.03.29; Accepted: 2011.04.22; Published: 2011.04.26

\begin{abstract}
This commentary highlights the findings by Kessinger et al. (Theranostics 20I I; I: 263-275) that dynamic $T_{2}{ }^{*}$-weighted magnetic resonance imaging (MRI) of cyclic RGD peptide-encoded superparamagnetic polymeric micelle (SPPM) nanoparticles allows quantitative analysis of tumor integrin $\alpha \mathrm{v} \beta 3$ expression, which can exclude the effect of blood volume and extravascular signal components and thus provide less biased tumor contrast and receptor specificity of probes.
\end{abstract}

Key words: Commentary; Superparamagnetic Nanoparticles; Integrin; Cyclic RGD Peptide; Dynamic MRI.

Targeted nanoprobes have made the field of molecular imaging an exciting arena with capabilities of delivering contrast agents to the desired site in vivo [1-3]. Targeted nanoprobes have been employed in a variety of imaging modalities such as positron emission tomography (PET), single photon emission computed tomography (SPECT), computed tomography $(\mathrm{CT})$, magnetic resonance imaging (MRI) and optical imaging [4-6]. Among the imaging modalities, MRI is a powerful medical diagnostic imaging technique for soft tissue imaging [7]. Magnetic nanoprobes have been around for a while in the field of MRI due to their exceptional spatial and temporal contrast enhancement [7-8]. Iron oxide nanoparticle (IONP) is one of the most adopted nanoprobes for $\mathrm{T}_{2}$ weighted MRI studies. Several IONP formulas have been approved for clinical application, for example, Ferumoxide (Endorem ${ }^{\circledR}$-Europe, Feridex ${ }^{\circledR}$ in the USA and Japan) and Ferucarbotran (Resovist ${ }^{\circledR}$-Europe and Japan) [9-13]. Ultra-small superparamagnetic iron oxide (USPIO) nanoparticles have shown potential in the imaging of molecular biomarkers such as integrins $[12,14-15]$. Integrins are heterodimeric transmembrane glycoproteins, a family of adhesion molecules which have been shown to play a major role in angiogenesis and tumor metastasis [16]. The $\alpha_{v} \beta_{3}$ integrin is significantly over-expressed on activated endothelial cells during angiogenesis but not on dormant endothelial cells. A salient feature of $\alpha_{v} \beta_{3}$ integrin is that it binds to extracellular matrix proteins via the three amino acid sequence arginine-glycine-aspartic acid (RGD). It is indicated that inhibition of integrin $\alpha_{v} \beta_{3}$ activity by cyclic RGD peptides, monoclonal antibodies and peptidomimetics may trigger endothelial cell apoptosis and result in inhibition of angiogenesis [17-18].

Data related to early events of binding and cellular uptake of targeted nanoprobes in cancer cells will reveal important insights on the critical factors that affect nanoprobe accumulation in vivo. In devel- 
oping new anti-angiogenic agents and for response evaluation, early time point kinetic studies are very important, where targeted nanoprobes can specifically bind to the cell surface receptors on the tumor endothelium from circulating blood. Gadolinium based $T_{1}$ based MRI contrast probes have been used for studying kinetic parameters using DCE-MRI [19-20]. Low molecular weight probes such as Gd-DTPA worked well in tumor perfusion studies and pharmacokinetic parameters were measured [21-22]. However, these probes are non-specific and require millimolar concentrations for detection, indicating lack of sensitivity. Developing sensitive and target specific nanoprobes for DCE-MRI would provide more reliable kinetic data.

In this volume of Theranostics Kessinger et al. [23] examined for the first time early time point kinetic studies of the binding events of targeted nanoprobes in vivo using time-resolved MRI. In this work, ultra-sensitive superparamagnetic polymeric micelle (SPPM) nanoprobes were employed to quantify the targeting kinetics of c(RGDfK) (cRGD)-encoded SPPM to angiogenic endothelium in subcutaneous lung, breast and glioblastoma xenograft models in mice using $\mathrm{T}_{2}{ }^{*}$-weighted HTR-MRI. A cluster of SPIO nanoparticles loaded into the core-shell of the polymeric micelle was used in the three different animal models. The nanoprobes were shown to increase the transverse relaxivity of the nanoprobes to over $400 \mathrm{Fe} \mathrm{mM}^{-1}$ $\mathrm{s}^{-1}$ at 7T, a 4-fold higher relaxivity compared to Feridex. $\mathrm{T}_{2}{ }^{*}$-weighted HTR-MRI with $1.3 \mathrm{~s}$ temporal resolution over $30 \mathrm{~min}$ shed light on the early phase of the targeting kinetics of $\mathrm{a}_{\mathrm{v}} \beta_{3}$-specific SPIO nanoprobes in different tumor xenografts models. SPIO nanoprobes conjugated with $\mathrm{a}_{\mathrm{v}} \beta_{3}$-specific cyclic RGDfK (cRGD) peptide and non-targeting cyclic RADfK (cRAD) as a control, were evaluated for difference in MR signal intensity (SI) over time. Comparison of the targeted and non-targeted SPPM data allowed for the subtraction of signal intensity contributions from blood concentration, clearance and enhanced permeability and retention (EPR) effect. Accumulation rates $\left(K_{a}\right)$ in the initial phase $(<5 \mathrm{~min})$ were found to be similar across all the three tumor models. However, a sustained accumulation of targeted cRGD-SPPM over $30 \mathrm{~min}$ was observed.

DCE-MRI is usually applied for characterization of abnormalities of perfusion, endothelial and epithelial permeability with $T_{1}$ weighted contrast imaging [24]. In this study, a new opportunity for characterizing the tumor specific targeting kinetics of nanoparticles by $\mathrm{T}_{2}$ weighted contrast imaging is reported and the initial results of $a_{v} \beta_{3}$ integrin receptor density quantification are achieved. Since the signal intensity curves of each region of interest (ROI) can be derived from $\mathrm{T}_{2}$ images, kinetic modeling analysis is able to estimate the pharmacokinetics of cRGD-SPPM contrast agent which reflects the tumor integrin level. Considering the non-specific binding of cRGD-SPPM via the leakage of contrast agent from capillary to the extravascular parenchymal space, they also studied the cRAD-SPPM as a non-targeting control. By subtracting the mean value of cRAD from cRGD curves, a compartment which only represents the specific cRGD concentration is achieved and relates to the specific accumulation rate independently.

As is the case with the two- or three-compartment kinetic modeling widely used in PET dynamic studies, one-compartment modeling from which non-targeting contrast agent is subtracted is also a direct method for receptor density study. The one-compartment model is easier to fit and more efficient for quantification of parameters; however, it is premised on accurate subtraction. For PET quantitative analysis of receptor density, the optimized model is comparable to the three compartment model for binding potential measurement.

In conclusion, this paper describes early time point kinetic binding studies of targeted nanoprobes that offer new opportunities in quantitative characterization of the targeting kinetics of cancer-specific nanoparticles to their intended biological targets in an intact animal. The data provide less biased tumor contrast and receptor specificity of probes and can exclude the effect of blood volume and extra-vascular signal components.

\section{Acknowledgment}

This work was supported by the intramural research program (IRP), National Institute of Biomedical Imaging and Bioengineering (NIBIB), National Institutes of Health (NIH).

\section{Conflict of Interest}

The authors have declared that no conflict of interest exists.

\section{References}

1. Lee JH, Huh YM, Jun YW, Seo JW, Jang JT, Song HT, et al. Artificially engineered magnetic nanoparticles for ultra-sensitive molecular imaging. Nat Med. 2007; 13: 95-9.

2. Popovtzer R, Agrawal A, Kotov NA, Popovtzer A, Balter J, Carey TE, et al. Targeted gold nanoparticles enable molecular CT imaging of cancer. Nano Lett. 2008; 8: 4593-6.

3. John R, Rezaeipoor R, Adie SG, Chaney EJ, Oldenburg AL, Marjanovic $\mathrm{M}$, et al. In vivo magnetomotive optical molecular imaging using targeted magnetic nanoprobes. Proc Natl Acad Sci U S A. 2010; 107: 8085-90.

4. Beer AJ, Kessler H, Wester H-J, Schwaiger M. PET imaging of integrin av $\beta 3$ expression. Theranostics. 2011; 1: 48-57. 
5. Zhou Y, Chakraborty S, Liu S. Radiolabeled cyclic RGD peptides as radiotracers for imaging tumors and thrombosis by SPECT. Theranostics. 2011; 1: 58-82.

6. Zhang Y, Yang Y, Cai W. Radiolabeled cyclic RGD peptides as radiotracers for imaging tumors and thrombosis by SPECT. Theranostics. 2011; 1: 58-82.

7. Jin Y, Jia C, Huang SW, O'Donnell M, Gao X. Multifunctional nanoparticles as coupled contrast agents. Nat Commun. 2010; 1 : 41.

8. Oghabian MA, Farahbakhsh NM. Potential use of nanoparticle based contrast agents in MRI: a molecular imaging perspective. J Biomed Nanotechnol. 2010; 6: 203-13.

9. Bhirde A, Xie J, Swierczewska M, Chen X. Nanoparticles for cell labeling. Nanoscale. 2011; 3: 142-53.

10. Xie J, Wang J, Niu G, Huang J, Chen K, Li X, et al. Human serum albumin coated iron oxide nanoparticles for efficient cell labeling. Chem Commun (Camb). 2010; 46: 433-5.

11. Xie J, Chen K, Huang J, Lee S, Wang J, Gao J, et al. PET/NIRF/MRI triple functional iron oxide nanoparticles. Biomaterials. 2010; 31: 3016-22.

12. Chen K, Xie J, Xu H, Behera D, Michalski MH, Biswal S, et al. Triblock copolymer coated iron oxide nanoparticle conjugate for tumor integrin targeting. Biomaterials. 2009; 30: 6912-9.

13. Lee HY, Li Z, Chen K, Hsu AR, Xu C, Xie J, et al. PET/MRI dual-modality tumor imaging using arginine-glycine-aspartic (RGD)-conjugated radiolabeled iron oxide nanoparticles. J Nucl Med. 2008; 49: 1371-9.

14. Xie J, Huang J, Li X, Sun S, Chen X. Iron oxide nanoparticle platform for biomedical applications. Curr Med Chem. 2009; 16: 1278-94.

15. Tan M, Lu Z-R. Integrin targeted MR imaging. Theranostics. 2011; 1: 83-101.

16. Niu $G$, Chen $X$. Why integrin as a primary target for imaging and therapy. Theranostics. 2011; 1: 30-47.

17. Millard M, Odde $S$, Neamati N. Integrin targeted therapeutics. Theranostics. 2011; 1: 154-88.

18. Chen K, Chen X. Integrin targeted delivery of chemotherapeutics. Theranostics. 2011; 1: 189-200.

19. Choyke PL, Dwyer AJ, Knopp MV. Functional tumor imaging with dynamic contrast-enhanced magnetic resonance imaging. J Magn Reson Imaging. 2003; 17: 509-20.

20. Turkbey B, Thomasson D, Pang Y, Bernardo M, Choyke PL. The role of dynamic contrast-enhanced MRI in cancer diagnosis and treatment. Diagn Interv Radiol. 2010; 16: 186-92.

21. Ahrens ET, Rothbacher U, Jacobs RE, Fraser SE. A model for MRI contrast enhancement using T1 agents. Proc Natl Acad Sci U S A. 1998; 95: 8443-8.

22. Su MY, Wang Z, Carpenter PM, Lao X, Muhler A, Nalcioglu O. Characterization of N-ethyl-N-nitrosourea-induced malignant and benign breast tumors in rats by using three MR contrast agents. J Magn Reson Imaging. 1999; 9: 177-86.

23. Kessinger CW, Togao O, Khemtong C, Huang G, Takahashi M, Gao J. Investigation of in vivo targeting kinetics of av $\beta 3$-specific superparamagnetic nanoprobes by high temporal resolution-MRI. Theranostics. 2011; 1:263-275.

24. Naish JH, Kershaw LE, Buckley DL, Jackson A, Waterton JC, Parker GJ. Modeling of contrast agent kinetics in the lung using T1-weighted dynamic contrast-enhanced MRI. Magn Reson Med. 2009; 61: 1507-14. 